\title{
ON THE RATE OF CONVERGENCE OF VISCOSITY SOLUTIONS FOR BOUNDARY VALUE PROBLEMS*
}

\author{
JENS LORENZ $\dagger$ AND RICHARD SANDERS $\ddagger$
}

\begin{abstract}
A class of singularly perturbed boundary value problems is considered for viscosity tending to zero. From compactness arguments it is known that the solutions converge to a limit function characterized by an entropy inequality. We formulate an approximate entropy inequality (AEI) and use it to obtain the order of convergence. The AEI is also used to obtain the order of convergence for monotone difference schemes.
\end{abstract}

Key words. singular perturbations, entropy inequality, bounded variation functions

AMS(MOS) subject classifications. Primary 34E99, 65L10

1. Introduction. In this paper we establish a minimal rate of convergence theorem for solutions to the singularly perturbed boundary value problem

$$
\begin{aligned}
& -\varepsilon \frac{d^{2}}{d x^{2}} u_{\varepsilon}+\frac{d}{d x} f\left(x, u_{\varepsilon}\right)+b\left(x, u_{\varepsilon}\right)=0, \\
& u_{\varepsilon}(0)=\gamma_{0}, \quad u_{\varepsilon}(0)=\gamma_{1},
\end{aligned}
$$

as the parameter $\varepsilon>0$ tends to zero. Throughout we impose no special conditions on $f(x, u)$ or $b(x, u)$ other than that they are smooth and satisfy

$$
\frac{\partial}{\partial u} b(x, u)-\left|\frac{\partial^{2}}{\partial u \partial x} f(x, u)\right| \geqq \mu>0
$$

for all $(x, u) \in[0,1] \times I$ where $I$ is an a priori interval determined from the maximum principle. ${ }^{1}$

It is well known that for positive $\varepsilon$, condition (1.2) implies that the boundary value problem (1.1) has a unique smooth solution; see [8], [9] for results in this direction.

As $\varepsilon$ tends to zero, solutions to (1.1) need not converge to a continuous function. Therefore, it is natural to seek a rate of convergence result in an integral sense. Below we show that there exists a function $\bar{u} \in B V$ such that for sufficiently small $\varepsilon$

$$
\int_{0}^{1}\left|u_{\varepsilon}-\bar{u}\right| d x \leqq \frac{C_{y}}{\mu} \sqrt{\varepsilon}
$$

where the constant $C_{\gamma}$ depends on the boundary data $\gamma_{0}$ and $\gamma_{1}$. In general the rate above is not valid unless condition (1.2) is imposed. That is to say there are examples

* Received by the editors January 13, 1986; accepted for publication May 8, 1986.

$\dagger$ Applied Mathematics 217-50, California Institute of Technology, Pasadena, California 91125. The research of this author was supported by National Science Foundation grant DMS 83-12264 and Department of Energy grant DE/AT/03/76ER/72012.

¥Department of Mathematics, University of Houston, Houston, Texas 77004. The research of this author was supported by National Science Foundation grant DMS 8505422.

${ }^{1}$ The interested reader can greatly relax the smoothness assumption we make on the coefficients of (1.1). We consider these details uninteresting however, and we take $f(x, u)$ and $b(x, u)$ as smooth as the specified number of derivatives indicate. 
of boundary value problems of the form (1.1) that violate (1.2) (they must satisfy an estimate like (1.2) with $\mu=0$ ) and satisfy

$$
\int_{0}^{1}\left|u_{\varepsilon}-\bar{u}\right| d x \geqq \text { const. } \varepsilon^{q}
$$

for any $\frac{1}{2} \geqq q>0$. Moreover, given that condition (1.2) is satisfied, the $L^{1}$ rate result (1.3) cannot be improved unless further conditions are imposed. That is, there are examples that satisfy condition (1.2) and satisfy

$$
\int_{0}^{1}\left|u_{\varepsilon}-\bar{u}\right| d x \geqq \text { const. } \sqrt{\varepsilon} \text {. }
$$

Some examples that demonstrate the sharpness of our rate result are presented at the end of this section.

The main contribution of this paper is to extend the techniques developed in [6], [13] to include problems with Dirichlet boundary conditions. The notion of an "approximate entropy inequality (AEI)," first introduced in the study of single conservation laws, is suitably modified to include boundary value problems of the type studied here. Specifically, what we show is that if a parameterized family of functions, say $\left\{v_{h}\right\}_{h>0}$, satisfies the uniform estimate

$$
\operatorname{var}\left(v_{h}\right) \leqq \text { const., }
$$

together with an $h$-dependent AEI, then $v_{h}$ satisfies (1.3) with $h$ taking the place of $\varepsilon$. We have intentionally been vague about the precise definition of the family $\left\{v_{h}\right\}_{h>0}$ since it is shown below that besides representing the family of solutions to (1.1) it can also represent a family of certain numerical approximations. In the application to numerical approximations $v_{h}$ denotes an interpolation of grid values generated by a finite difference scheme, and $h$ denotes a measure of grid refinement.

In $\S 2$ the characterizing "entropy inequality" for the limit of solutions to (1.1) is stated; see [2], [3], [5], [14] for a thorough development of these ideas. The "approximate entropy inequality" is also defined in this section, and solutions of (1.1) are shown to satisfy it. The abstract rate of convergence theorem implied by the AEI is also stated in $\S 2$. In $\S 3$ the abstract rate of convergence theorem, stated in $\S 2$, is proved. Finally in $\S 4$ the rate of convergence theorem is applied to numerical approximations generated by certain types of finite difference schemes.

We should mention that most of the results of this paper can be routinely extended to quasilinear Dirichlet problems in many space dimensions. This will be the topic of future work; see [12], where somewhat parallel techniques are applied to nonlinear problems with boundary conditions of Neumann type.

We conclude this section by constructing some nontrivial examples of the type mentioned above. First we show that if (1.2) is violated then an arbitrarily slow $L^{1}$ rate of convergence is possible. To this end, consider the boundary value problem

$$
\begin{aligned}
& -\varepsilon \frac{d^{2}}{d x^{2}} u_{\varepsilon}+\frac{d}{d x}\left(\gamma-u_{\varepsilon}\right)^{2 p}=0, \\
& u_{\varepsilon}(0)=0, \quad u_{\varepsilon}(1)=\gamma>0,
\end{aligned}
$$

where we take $p>1$. Clearly (1.4) violates (1.2). By [8, Thm. 4] the solutions of (1.4) tend uniformly to $\gamma$ on any interval $[\delta, 1], 1>\delta>0$, as $\varepsilon$ tends to zero. Therefore, we wish to examine

$$
\int_{0}^{1}\left|u_{\varepsilon}-\gamma\right| d x
$$


With this end in mind we first consider

$$
\begin{aligned}
& -\varepsilon \frac{d^{2}}{d x^{2}} v_{\varepsilon}+\frac{d}{d x}\left(\gamma-v_{\varepsilon}\right)^{2 p}=0, \\
& v_{\varepsilon}(0)=0, \quad v_{\varepsilon}(\infty)=\gamma,
\end{aligned}
$$

which can be integrated exactly, giving

$$
v_{\varepsilon}(x)=\Phi^{-1}(x / \varepsilon),
$$

where

$$
\Phi(w)=\int_{0}^{w}(\gamma-s)^{-2 p} d s
$$

Since $v_{\varepsilon}(x)+\gamma-v_{\varepsilon}(1)$ is an upper solution for (1.4), it follows that this function is $\geqq u_{\varepsilon}(x)$; thus

$$
\gamma-u_{\varepsilon}(x) \geqq v_{\varepsilon}(1)-v_{\varepsilon}(x) \geqq 0,
$$

which implies

$$
\int_{0}^{1}\left|u_{\varepsilon}-\gamma\right| d x \geqq \int_{0}^{1}\left(v_{\varepsilon}(1)-v_{\varepsilon}(x)\right) d x .
$$

Interchanging the order of integration and using (1.7), we have that the right-hand side of $(1.8)$ is given by

$$
\varepsilon \int_{0}^{v_{\varepsilon}(1)} \Phi(w) d w
$$

Finally, a simple calculation will reveal that

$$
\varepsilon \int_{0}^{v_{\varepsilon}(1)} \Phi(w) d w=\frac{1}{2 p-2}\left(\frac{\varepsilon}{2 p-1}\right)^{1 / 2 p-1}+O(\varepsilon),
$$

which therefore shows that

$$
\int_{0}^{1}\left|u_{\varepsilon}-\gamma\right| d x \geqq C_{p}\left(\varepsilon^{1 / 2 p-1}\right),
$$

as $\varepsilon$ tends to zero.

To establish the fact, given only condition (1.2), our rate result (1.3) is the best possible, we note that the trivial example

$$
\begin{aligned}
& -\varepsilon \frac{d^{2}}{d x^{2}} u_{\varepsilon}+u_{\varepsilon}=0, \\
& u_{\varepsilon}(0)=0, \quad u_{\varepsilon}(1)=1,
\end{aligned}
$$

satisfies the $\sqrt{\varepsilon} L^{1}$ rate of convergence exactly. A less trivial example is given by

$$
\begin{aligned}
& -\varepsilon \frac{d^{2}}{d x^{2}} u_{\varepsilon}+\frac{d}{d x}\left((1-x) u_{\varepsilon}\right)+2 u_{\varepsilon}=0, \\
& u_{\varepsilon}(0)=0, \quad u_{\varepsilon}(1)=1 .
\end{aligned}
$$

To obtain the sharp $\sqrt{\varepsilon}$ rate for example (1.9) we apply the "shooting method." (Although this method is less general than the techniques we present in the following 
sections, it takes into account specific properties of $f(x, u)$ and often leads to sharper results than ours; see [4] for applications of differential inequalities.) From our results below, we expect that $\lim _{\varepsilon \downarrow 0} u_{\varepsilon}=0$. The maximum principle together with integrating (1.9) gives us that

$$
\int_{0}^{1}\left|u_{\varepsilon}-0\right| d x=\frac{\varepsilon}{2}\left[\frac{d}{d x} u_{\varepsilon}(1)-\frac{d}{d x} u_{\varepsilon}(0)\right] .
$$

By applying the shooting method, it is easy to conclude that for all $\varepsilon>0$ sufficiently small, we have

$$
\frac{1}{2 \sqrt{\varepsilon}} \leqq \frac{d}{d x} u_{\varepsilon}(1) \leqq \frac{3}{\sqrt{\varepsilon}}
$$

and

$$
0 \leqq \frac{d}{d x} u_{\varepsilon}(0) \leqq 1
$$

Inserting these inequalities into (1.10) we finally get

$$
\frac{\sqrt{\varepsilon}}{4}-O(\varepsilon) \leqq \int_{0}^{1}\left|u_{\varepsilon}-0\right| d x \leqq \frac{3}{2} \sqrt{\varepsilon},
$$

which establishes our $L^{1}$ rate for example (1.9).

2. The approximate entropy inequality. Throughout this paper the following notation is used:

(i) $B V$ denotes the space of functions $u:[0,1] \rightarrow \mathbb{R}$ of bounded variation.

(ii) $\operatorname{var}(u)$ is the total variation of $u \in B V$.

(iii) $C_{+}^{\infty}$ denotes the space of functions $\phi: \mathbb{R} \rightarrow \mathbb{R}$ which are infinitely differentiable and nonnegative.

The sign-function is defined by

$$
\operatorname{sgn}(u)= \begin{cases}-1 & \text { if } u<0 \\ 0 & \text { if } u=0 \\ 1 & \text { if } u>0\end{cases}
$$

and

$$
\operatorname{sgn}_{\delta}(u)= \begin{cases}u /|u| & \text { for }|u| \geqq \delta, \\ u / \delta & \text { for }|u|<\delta\end{cases}
$$

denotes a Lipschitz continuous approximation to $\operatorname{sgn}(u)$ for $\delta>0$. We furthermore use

$$
\begin{aligned}
& \|u\|_{\infty}=\sup \{|u(x)|: 0 \leqq x \leqq 1\} \quad \text { for } u \in L^{\infty}[0,1], \\
& \|u\|_{1}=\int_{0}^{1}|u(x)| d x \text { for } u \in L^{1}[0,1] .
\end{aligned}
$$

For simplicity we assume that $f(x, u) \in C^{2}([0,1] \times \mathbb{R})$ and $b(x, u) \in C^{1}([0,1] \times \mathbb{R})$ although we recognize that weaker conditions are sufficient. The essential assumption is nevertheless condition (1.2), and it will be assumed throughout.

In the next proposition we state some known results concerning the second order boundary value problem (1.1); see [2], [9]. These facts are relevant in what follows. 
Proposition 2.1. For all $\varepsilon>0,(1.1)$ has a unique smooth solution $u_{\varepsilon}$. Moreover, there exists a constant $c$, not depending on $\varepsilon$, such that

$$
\left\|u_{\varepsilon}\right\|_{\infty}+\operatorname{var}\left(u_{\varepsilon}\right) \leqq c .
$$

Finally, there exists a unique (a.e.) function $\bar{u} \in B V$ such that

$$
\left\|u_{\varepsilon}-\bar{u}\right\|_{1} \rightarrow 0 \text {, }
$$

as $\varepsilon \downarrow 0$.

It is known that the limit $\bar{u} \in B V$ is the only (a.e.) $B V$ function satisfying the following so-called "entropy inequality":

For all $k \in \mathbb{R}$ and all $\phi \in C_{+}^{\infty}$

$$
\begin{aligned}
& \int_{0}^{1} \operatorname{sgn}(\bar{u}-k)\left\{-(f(x, \bar{u})-f(x, k)) \phi_{x}+\left(b(x, \bar{u})+\frac{\partial}{\partial x} f(x, k)\right) \phi\right\} d x \\
& \quad+\operatorname{sgn}\left(\gamma_{1}-k\right)\{f(1, \bar{u}(1-))-f(1, k)\} \phi(1) \\
& \quad-\operatorname{sgn}\left(\gamma_{0}-k\right)\{f(0, \bar{u}(0+))-f(0, k)\} \phi(0) \leqq 0 .
\end{aligned}
$$

We now state what we call the "approximate entropy inequality" (or AEI) for a parameterized family of $B V$ functions $\left\{v_{h}\right\}_{0<h \leqq h_{0}}$. Below the family of solutions to (1.1) are shown to satisfy the AEI, and in $\S 4$ certain numerical approximations are shown to satisfy the AEI as well.

Definition 2.1. A family of $B V$ functions $\left\{v_{h}\right\}_{0<h \leqq h_{0}}$ is said to satisfy the AEI if there exists nonnegative functions

$$
R_{h}^{0}, \quad R_{h}^{1}, \quad R_{h}^{2} \in B V, \quad 0<h \leqq h_{0},
$$

with the properties that

A. There exists a constant $c$ independent of $0<h \leqq h_{0}$ and $0 \leqq \delta \leqq 1$ such that

$$
\begin{array}{ll}
\text { A0. } & \int_{0}^{\delta} R_{h}^{0}(s) d s \leqq c\left(h+\delta^{2}\right), \\
\text { A1. } & \int_{1-\delta}^{1} R_{h}^{1}(s) d s \leqq c\left(h+\delta^{2}\right), \\
\text { A2. } & \int_{0}^{1} R_{h}^{2}(s) d s \leqq c h ;
\end{array}
$$

B. For all $k \in \mathbb{R}$, all $\phi \in C_{+}^{\infty}$ and almost every $\alpha, \beta \in(0,1)$ we have

$$
\begin{aligned}
\int_{0}^{1} \operatorname{sgn}\left(v_{h}-k\right) & \left\{-\left(f\left(x, v_{h}\right)-f(x, k)\right) \phi_{x}+\left(b\left(x, v_{h}\right)+\frac{\partial}{\partial x} f(x, k)\right) \phi\right\} d x \\
& +\operatorname{sgn}\left(\gamma_{1}-k\right)\left\{f\left(\beta, v_{h}(\beta)\right)-f(1, k)\right\} \phi(1) \\
& -\operatorname{sgn}\left(\gamma_{0}-k\right)\left\{f\left(\alpha, v_{h}(\alpha)\right)-f(0, k)\right\} \phi(0) \\
\leqq & R_{h}^{1}(\beta) \phi(1)+R_{h}^{0}(\alpha) \phi(0)+\int_{0}^{1} R_{h}^{2}\left|\phi_{x}\right| d x .
\end{aligned}
$$

In $\S 3$ we prove the following theorem.

THEOREM 2.1. Let $\left\{v_{h}\right\}$ satisfy the AEI and assume that there exists a constant $C_{1}$ independent of $0<h \leqq h_{0}$ such that $\left\|v_{h}\right\|_{\infty}+\operatorname{var}\left(v_{h}\right) \leqq C_{1}$. Then, there exists a constant $C_{2}$ independent of $0<h \leqq h_{0}$ such that

$$
\left\|v_{h}-\bar{u}\right\|_{1} \leqq C_{2} \sqrt{h},
$$

where $\bar{u}=\lim _{\varepsilon \downarrow 0} u_{\varepsilon}$. 
The result of Theorem 2.1 applies directly to the family of solutions to (1.1).

COROLLARY. The family $\left\{u_{\varepsilon}\right\}_{\varepsilon>0}$ of solutions to (1.1) satisfies the AEI; consequently we have the estimate

$$
\left\|u_{\varepsilon}-\bar{u}\right\| \leqq C \sqrt{\varepsilon} .
$$

Proof. Multiply the identity

$$
0=-\varepsilon \frac{d^{2}}{d x^{2}} u_{\varepsilon}+\frac{d}{d x}\left(f\left(x, u_{\varepsilon}\right)-f(x, k)\right)+\left(b\left(x, u_{\varepsilon}\right)+\frac{\partial}{\partial x} f(x, k)\right)
$$

by $\operatorname{sgn}_{\delta}\left(u_{\varepsilon}-k\right) \phi, \phi \in C_{+}^{\infty}$, integrate over $0 \leqq x \leqq 1$, apply integration by parts, use the estimate

$$
\int_{0}^{1}\left(\frac{d}{d x} \operatorname{sgn}_{\delta}\left(u_{\varepsilon}-k\right) \frac{d}{d x} u_{\varepsilon}\right) \phi d x \geqq 0
$$

and let $\delta \downarrow 0$ to find with Lebesgue's dominated convergence theorem that

$$
\begin{aligned}
\int_{0}^{1} \operatorname{sgn}\left(u_{\varepsilon}-k\right) & \left\{-\left(f\left(x, u_{\varepsilon}\right)-f(x, k)\right) \phi_{x}+\left(b\left(x, u_{\varepsilon}\right)+\frac{\partial}{\partial x} f(x, k)\right) \phi\right\} d x \\
& +\operatorname{sgn}\left(\gamma_{1}-k\right)\left\{f\left(1, \gamma_{1}\right)-f(1, k)-\varepsilon \frac{d}{d x} u_{\varepsilon}(1)\right\} \phi(1) \\
& -\operatorname{sgn}\left(\gamma_{0}-k\right)\left\{f\left(0, \gamma_{0}\right)-f(0, k)-\varepsilon \frac{d}{d x} u_{\varepsilon}(0)\right\} \phi(0) \\
\leqq & \varepsilon \int_{0}^{1} \operatorname{sgn}\left(u_{\varepsilon}-k\right)\left(\frac{d}{d x} u_{\varepsilon}\right) \phi_{x} d x .
\end{aligned}
$$

Integrating the differential equation (1.1) we make it evident that the boundary terms above can be written as

$$
\operatorname{sgn}\left(\gamma_{1}-k\right)\left\{\left(f\left(\beta, u_{\varepsilon}(\beta)\right)-f(1, k)\right)-\varepsilon \frac{d u_{\varepsilon}}{d x}(\beta)-\int_{\beta}^{1} b\left(x, u_{\varepsilon}\right) d x\right\} \phi(1),
$$

and

$$
\operatorname{sgn}\left(\gamma_{0}-k\right)\left\{\left(f\left(\alpha, u_{\varepsilon}(\alpha)\right)-f(0, k)\right)-\varepsilon \frac{d u_{\varepsilon}}{d x}(\alpha)+\int_{0}^{\alpha} b\left(x, u_{\varepsilon}\right) d x\right\} \phi(0) .
$$

Inserting these identities into the inequality above we easily find that $u_{\varepsilon}$ satisfies the AEI with

$$
\begin{aligned}
& R_{\varepsilon}^{0}(\alpha)=\varepsilon\left|\frac{d}{d x} u_{\varepsilon}(\alpha)\right|+\int_{0}^{\alpha}\left|b\left(x, u_{\varepsilon}\right)\right| d x, \\
& R_{\varepsilon}^{1}(\beta)=\varepsilon\left|\frac{d}{d x} u_{\varepsilon}(\beta)\right|+\int_{\beta}^{1}\left|b\left(x, u_{\varepsilon}\right)\right| d x, \\
& R_{\varepsilon}^{2}(x)=\varepsilon\left|\frac{d}{d x} u_{\varepsilon}(x)\right|
\end{aligned}
$$

Using the result of Proposition 2.1 we finally conclude that $R_{\varepsilon}^{0}, R_{\varepsilon}^{1}$ and $R_{\varepsilon}^{2}$ above satisfy properties A0, A1 and A2 of Definition 2.1 with $\varepsilon$ taking the role of $h$. 
3. Proof of Theorem 2.1. Let $\left\{u_{\varepsilon}\right\}_{\varepsilon>0}$ denote the family of solutions to (1.1), and let $\left\{v_{h}\right\}_{h>0}$ denote a family of functions which have uniformly bounded variation and which satisfy the AEI. Moreover, let $R_{\varepsilon}^{j}$, (resp. $R_{h}^{j}$ ), with $j=0,1,2$, represent the estimating functions of the AEI of Definition 2.1 for $\left\{u_{\varepsilon}\right\}_{\varepsilon>0}$, (resp. $\left\{v_{h}\right\}_{h>0}$ ). The proof of Theorem 2.1 is essentially a test function argument with a particular family of test functions $\phi_{\delta}$ of the form

$$
\phi_{\delta}(x)=\frac{1}{\delta} \phi\left(\frac{x}{\delta}\right)
$$

where $\phi \in C_{+}^{\infty}$ is symmetric, $\int_{-\infty}^{\infty} \phi(x) d x=1$ and supp $(\phi) \subset(-1,1)$. Now consider the AEI of Definition 2.1 applied to $u_{\varepsilon}(x)$ with $k=v_{h}(y)$ and the test function $\phi$ replaced by $\phi(x)=\phi_{\delta}(x-y)$ and with $\alpha=\beta=y$ in $R_{h}^{j}, j=0,1$. Integrate the resulting inequality from $y=0$ to $y=1$. Since $v_{h}$ also satisfies the AEI, the same procedure can be done as above with the roles of $v_{h}$ and $u_{\varepsilon}$ reversed. Adding both resulting inequalities together we obtain

$\int_{0}^{1} \int_{0}^{1} \operatorname{sgn}\left(u_{\varepsilon}(x)-v_{h}(y)\right)\left\{\left(b\left(x, u_{\varepsilon}(x)\right)-b\left(y, v_{h}(y)\right)\right)\right.$

$$
\begin{aligned}
& \left.+\left(\frac{\partial}{\partial x} f\left(x, v_{h}(y)\right)-\frac{\partial}{\partial y} f\left(y, u_{\varepsilon}(x)\right)\right)\right\} \phi_{\delta}(x-y) d x d y \\
& +\int_{0}^{1} \int_{0}^{1} \operatorname{sgn}\left(( u _ { \varepsilon } ( x ) - v _ { h } ( y ) ) \left\{\left(f\left(x, v_{h}(y)\right)-f\left(y, v_{h}(y)\right)\right)\right.\right.
\end{aligned}
$$

$$
\begin{aligned}
& \left.+\left(f\left(y, u_{\varepsilon}(x)\right)-f\left(x, u_{\varepsilon}(x)\right)\right)\right\} \frac{d}{d x} \phi_{\delta}(x-y) d x d y+T_{1}(\varepsilon, h, \delta)-T_{0}(\varepsilon, h, \delta) \\
\leqq & P_{h}^{0}(\delta)+P_{h}^{1}(\delta)+P_{h}^{2}(\delta)+P_{\varepsilon}^{0}(\delta)+P_{\varepsilon}^{1}(\delta)+P_{\varepsilon}^{2}(\delta),
\end{aligned}
$$

where

$$
\begin{aligned}
T_{1}(\varepsilon, h, \delta)=\int_{0}^{1} & \operatorname{sgn}\left(\gamma_{1}-v_{h}(y)\right)\left\{f\left(y, u_{\varepsilon}(y)\right)-f\left(1, v_{h}(y)\right)\right\} \phi_{\delta}(1-y) d y \\
& \quad+\int_{0}^{1} \operatorname{sgn}\left(\gamma_{1}-u_{\varepsilon}(x)\right)\left\{f\left(x, v_{h}(x)\right)-f\left(1, u_{\varepsilon}(x)\right)\right\} \phi_{\delta}(1-x) d x,
\end{aligned}
$$

$$
\begin{aligned}
T_{0}(\varepsilon, h, \delta)=\int_{0}^{1} & \operatorname{sgn}\left(\gamma_{0}-v_{h}(y)\right)\left\{f\left(y, u_{\varepsilon}(y)\right)-f\left(0, v_{h}(y)\right)\right\} \phi_{\delta}(y) d y \\
& +\int_{0}^{1} \operatorname{sgn}\left(\gamma_{0}-u_{\varepsilon}(x)\right)\left\{f\left(x, v_{h}(x)\right)-f\left(0, u_{\varepsilon}(x)\right)\right\} \phi_{\delta}(x) d x,
\end{aligned}
$$

and

$$
\begin{aligned}
& P_{h}^{0}(\delta)=\int_{0}^{1} R_{h}^{0}(x) \phi_{\delta}(x) d x, \\
& P_{h}^{1}(\delta)=\int_{0}^{1} R_{h}^{1}(x) \phi_{\delta}(1-x) d x, \\
& P_{h}^{2}(\delta)=\int_{0}^{1} \int_{0}^{1} R_{h}^{2}(y)\left|\frac{d}{d x} \phi_{\delta}(x-y)\right| d x d y,
\end{aligned}
$$

and a similar expression for $P_{\varepsilon}^{j}(\delta)$. The proof is divided into basically the following four lemmas. 
LEMMA 3.1. There is a constant $c$ independent of $\varepsilon>0, h>0$, and $\delta>0$ such that we have

$$
\text { r.h.s. } \leqq c\left(\frac{\varepsilon}{\delta}+\frac{h}{\delta}+\delta\right),
$$

where r.h.s. is the right-hand side of (3.1).

LEMMA 3.2. There is another constant $c$ as above such that we have

$$
T_{0}(\varepsilon, h, \delta) \leqq c\left(\frac{\varepsilon}{\delta}+\frac{h}{\delta}+\delta\right), \quad-T_{1}(\varepsilon, h, \delta) \leqq c\left(\frac{\varepsilon}{\delta}+\frac{h}{\delta}+\delta\right),
$$

where $T_{0}$ and $T_{1}$ are defined in (3.2), (3.3).

LEMMA 3.3. There is a constant $c$ independent of positive $\varepsilon, h$ and $\delta$ such that

$$
\begin{aligned}
& \mid \int_{0}^{1} \int_{0}^{1} \operatorname{sgn}\left(u_{\varepsilon}(x)-v_{h}(y)\right)\left\{\left(f\left(x, v_{h}(y)\right)-f\left(y, v_{h}(y)\right)\right)\right. \\
& \left.\quad+\left(f\left(y, u_{\varepsilon}(x)\right)-f\left(x, u_{\varepsilon}(x)\right)\right)\right\} \frac{d}{d x} \phi_{\delta}(x-y) d x d y \mid \leqq c \delta .
\end{aligned}
$$

LEMMA 3.4. There is a constant $c$ independent of positive $\varepsilon, h, \delta \leqq 1 / 2$ such that

$$
\begin{aligned}
\int_{0}^{1} \mid\left(b\left(x, u_{\varepsilon}(x)\right)-\frac{\partial}{\partial x} f\left(x, u_{\varepsilon}(x)\right)\right) & -\left(b\left(x, v_{h}(x)\right)-\frac{\partial}{\partial x} f\left(x, v_{h}(x)\right)\right) \mid d x \\
\leqq 2 \int_{0}^{1} \int_{0}^{1} \operatorname{sgn}\left(u_{\varepsilon}(x)-v_{h}(y)\right) & \left\{\left(b\left(x, u_{\varepsilon}(x)\right)-b\left(y, v_{h}(y)\right)\right)\right. \\
+ & \left.\left(\frac{\partial}{\partial x} f\left(x, v_{h}(y)\right)-\frac{\partial}{\partial y} f\left(y, u_{\varepsilon}(x)\right)\right)\right\} \phi_{\delta}(x-y) d x d y+c \delta .
\end{aligned}
$$

Given the results above, the final result follows by first noting that, along with condition (1.2), they imply

$$
\begin{aligned}
\mu \int_{0}^{1} \mid & u_{\varepsilon}(x)-v_{h}(x) \mid d x \\
& \leqq \int_{0}^{1}\left|\left(b\left(x, u_{\varepsilon}(x)\right)-\frac{\partial}{\partial x} f\left(x, u_{\varepsilon}(x)\right)\right)-\left(b\left(x, v_{h}(x)\right)-\frac{\partial}{\partial x} f\left(x, v_{h}(x)\right)\right)\right| d x \\
& \leqq \hat{c}\left(\frac{\varepsilon}{\delta}+\frac{h}{\delta}+\delta\right)
\end{aligned}
$$

with $\hat{c}$ independent of positive $\varepsilon, h$ and $\delta \leqq \frac{1}{2}$. Sending $\varepsilon$ to zero, we conclude that

$$
\left\|\bar{u}-v_{h}\right\|_{1} \leqq \frac{\hat{c}}{\mu}\left(\frac{h}{\delta}+\delta\right),
$$

and choosing $\delta=\sqrt{h}$ proves the theorem.

Proof of Lemma 3.1. The terms to be estimated are given in (3.4)-(3.6). Note that

$$
P_{h}^{0}(\delta)=\int_{0}^{\delta} R_{h}^{0}(x) \phi_{\delta}(x) d x \leqq \frac{C}{\delta}\left(h+\delta^{2}\right)
$$

and

$$
P_{h}^{2}(\delta)=\int_{0}^{1} R_{h}^{2}(y)\left\{\int_{0}^{1}\left|\frac{d}{d x} \phi_{\delta}(y-x)\right| d x\right\} d y \leqq C \frac{h}{\delta}
$$


where the first inequality above follows from the definition $R_{h}^{0}(x)$ and $\phi_{\delta}(x)$, and the second follows from the definition of $R_{h}^{2}(y)$ and the fact that

$$
\int_{0}^{1}\left|\frac{d}{d x} \phi_{\delta}(y-x)\right| d x \leqq \frac{1}{\delta^{2}} \int_{-\infty}^{\infty}\left|\phi^{\prime}\left(\frac{z}{\delta}\right)\right| d z \leqq C / \delta .
$$

Similar estimates hold for the remaining terms on the right-hand side of (3.1).

Proof of Lemma 3.2. We only estimate $T_{0}$ since $-T_{1}$ can be treated similarly. Note that $T_{0}$ can be written as

$$
\begin{aligned}
T_{0}(\varepsilon, h, \delta)=\int_{0}^{1} & \left\{\operatorname{sgn}\left(\gamma_{0}-v_{h}\right)-\operatorname{sgn}\left(\gamma_{0}-u_{\varepsilon}\right)\right\} \\
& \cdot\left\{\left(f\left(x, u_{\varepsilon}\right)-f\left(x, \gamma_{0}\right)\right)+\left(f\left(x, \gamma_{0}\right)-f\left(x, v_{h}\right)\right)\right\} \phi_{\delta}(x) d x \\
& +\int_{0}^{1} \operatorname{sgn}\left(\gamma_{0}-v_{h}\right)\left\{f\left(x, v_{h}\right)-f\left(0, v_{h}\right)\right\} \phi_{\delta}(x) d x \\
& +\int_{0}^{1} \operatorname{sgn}\left(\gamma_{0}-u_{\varepsilon}\right)\left\{f\left(x, u_{\varepsilon}\right)-f\left(0, u_{\varepsilon}\right)\right\} \phi_{\delta}(x) d x .
\end{aligned}
$$

Clearly, the second two terms above can be bounded above by $C \delta$. The first term above is bounded above by

$$
\begin{aligned}
& \int_{0}^{1}\left[\left|f\left(x, u_{\varepsilon}\right)-f\left(x, \gamma_{0}\right)\right|+\operatorname{sgn}\left(u_{\varepsilon}-\gamma_{0}\right)\left(f\left(x, u_{\varepsilon}\right)-f\left(x, \gamma_{0}\right)\right)\right] \phi_{\delta}(x) d x \\
& \quad+\int_{0}^{1}\left[\left|f\left(x, v_{h}\right)-f\left(x, \gamma_{0}\right)\right|+\operatorname{sgn}\left(v_{h}-\gamma_{0}\right)\left(f\left(x, v_{h}\right)-f\left(x, \gamma_{0}\right)\right)\right] \phi_{\delta}(x) d x .
\end{aligned}
$$

We estimate the first integral above only since the second integral can be estimated similarly. Return now to the AEI applied to $u_{\varepsilon}$, and set $k=\gamma_{0}$ and suppose there we replace the test function $\phi(x)$ with a test function approaching

$$
H\left(x-x_{0}\right)= \begin{cases}1, & x<x_{0}, \\ 0, & x \geqq x_{0} .\end{cases}
$$

Doing so we find that for almost every $x_{0} \in(0,1)$ the AEI implies that

$$
\begin{aligned}
\operatorname{sgn}\left(u_{\varepsilon}\left(x_{0}\right)-\gamma_{0}\right)\left\{f\left(x_{0}, u_{\varepsilon}\left(x_{0}\right)\right)-f\left(x_{0}, \gamma_{0}\right)\right\} & \leqq R_{\varepsilon}^{0}\left(x_{0}\right)+R_{\varepsilon}^{2}\left(x_{0}\right)+c x_{0} \\
& \equiv e_{\varepsilon}\left(x_{0}\right) .
\end{aligned}
$$

To find an estimate for the integral in question, we note the obvious implication: If $Q \leqq e$ then $|Q|+Q \leqq 2 e$. Therefore, applying (3.8) (and the analogous estimate for $v_{h}$ ) to (3.7) shows that (3.7) is bounded above by

$$
2 \int_{0}^{1}\left[e_{\varepsilon}(x)+e_{h}(x)\right] \phi_{\delta}(x) d x .
$$

Finally, applying the simple estimates of the previous lemma completes the proof of the present lemma.

The proof of Lemma 3.3 is routine and is left to the reader.

Proof of Lemma 3.4. The proof of this lemma can be given for fixed $\varepsilon>0, h>0$, using only condition (1.2) and the uniform estimate

$$
\left\|u_{\varepsilon}\right\|_{\infty}+\int_{0}^{1}\left|\frac{d}{d x} u_{\varepsilon}\right| d x \leqq C_{0} .
$$


For convenience we omit the subscripts $\varepsilon$ and $h$. Condition (1.2) and a simple rearrangement gives us that

$$
\begin{aligned}
& \mid\left(b(y, u(y))-\frac{\partial}{\partial y} f(y, u(y))\right)-\left(b(y, v(y))-\frac{\partial}{\partial y} f(y, v(y))\right) \mid \\
& \leqq \operatorname{sgn}(u(x)-v(y))\{(b(x, u(x))-b(y, v(y))) \\
&\left.+\left(\frac{\partial}{\partial x} f(x, v(y))-\frac{\partial}{\partial y} f(y, u(x))\right)\right\} \\
&+L_{1}|x-y|+L_{2}|u(x)-u(y)|,
\end{aligned}
$$

where

$$
\begin{aligned}
& L_{1}=\max \left\{\left|\frac{\partial}{\partial x} b(x, u)\right|+\left|\frac{\partial^{2}}{\partial x^{2}} f(x, u)\right|, u \in I, x \in[0,1]\right\}, \\
& L_{2}=\max \left\{\left|\frac{\partial}{\partial u} b(x, u)\right|+\left|\frac{\partial^{2}}{\partial x \partial u} f(x, u)\right|, u \in I, x \in[0,1]\right\} .
\end{aligned}
$$

Furthermore, it is obvious that for all $0 \leqq y \leqq 1$ and all $\frac{1}{2} \geqq \delta>0$

$$
\int_{0}^{1} \phi_{\delta}(x-y) d x \geqq \frac{1}{2} \text {. }
$$

Therefore

$$
\begin{aligned}
& \int_{0}^{1}\left|\left(b(y, u(y))-\frac{\partial}{\partial y} f(y, u(y))\right)-\left(b(y, v(y))-\frac{\partial}{\partial y} f(y, v(y))\right)\right| d y \\
& \leqq L_{1} \int_{0}^{1} \int_{0}^{1}|x-y| \phi_{\delta}(x-y) d x d y+2 L_{2} \int_{0}^{1} \int_{0}^{1}|u(x)-u(y)| \phi_{\delta}(x-y) d x d y \\
&+2 \int_{0}^{1} \int_{0}^{1} \operatorname{sgn}(u(x)-v(y)) \\
& \cdot\left\{(b(x, u(x))-b(y, v(y)))+\left(\frac{\partial}{\partial x} f(x, v(y))-\frac{\partial}{\partial y} f(y, u(x))\right)\right\} \phi_{\delta}(x-y) d x d y .
\end{aligned}
$$

To complete the proof we need only estimate the second term on the right-hand side above since the other terms have been dealt with already. To see that

$$
\int_{0}^{1} \int_{0}^{1}|u(x)-u(y)| \phi_{\delta}(x-y) d x d y \leqq C \delta,
$$

extend the smooth function $u(x)$ to the whole real line by $u(x)=u(0)$ for $x<0$ and $u(x)=u(1)$ for $x>1$. Then for each $0 \leqq y \leqq 1$ we have

$$
\begin{aligned}
\int_{0}^{1}|u(x)-u(y)| \phi_{\delta}(x-y) d x & \leqq \int_{y-\delta}^{y+\delta} \int_{y-\delta}^{y+\delta}\left|u^{\prime}(s)\right| \phi_{\delta}(x-y) d x d s \\
& =\int_{-\delta}^{\delta}\left|u^{\prime}(y+s)\right| d s .
\end{aligned}
$$


Integrating this inequality with respect to $y$ and interchanging the order of integration makes the desired result obvious.

4. Application to difference schemes. In this section we give another application of the AEI. We show that certain finite difference schemes yield approximations that satisfy the AEI; hence, according to Theorem 2.1 , they satisfy the $\sqrt{h} L^{1}$ rate of convergence. We begin with a few preliminaries.

Partition the interval $[0,1]$ into subintervals $I_{j}=\left[x_{j}, x_{j+1}\right], 0 \leqq j \leqq J-1$, with $x_{0}=0$ and $x_{J}=1$, and define $\Delta x_{j}=\left(x_{j+1}-x_{j}\right)$. Define the approximate solution $v_{h}$ by

$$
v_{h}(x)=\sum_{j=0}^{J-1} u_{j} \chi_{I_{j}}(x),
$$

where $\chi_{I_{j}}$ is the characteristic function of the interval $I_{j}$. We shall consider a class of finite difference schemes of the form

$$
\begin{aligned}
& \Delta^{+} F\left(x_{j}, u_{j}, u_{j-1}\right)+\Delta x_{j} B\left(x_{j}, x_{j+1}, u_{j}\right)=0, \quad 0 \leqq j \leqq J-1, \\
& u_{-1}=\gamma_{0}, \quad u_{J}=\gamma_{1},
\end{aligned}
$$

where the forward difference operator $\Delta^{+}$is defined by $\Delta^{+} \alpha_{j}=\alpha_{j+1}-\alpha_{j}$ and $B\left(x_{j}, x_{j+1}, u_{j}\right)$ is given by

$$
B\left(x_{j}, x_{j+1}, u\right)=b\left(x_{j}, u\right)+\frac{\partial}{\partial x} f\left(x_{j}, u\right)-\frac{1}{\Delta x_{j}}\left(f\left(x_{j+1}, u\right)-f\left(x_{j}, u\right)\right) .
$$

The numerical flux function $F(\cdot, \cdot, \cdot)$ of $(4.1)$ is assumed throughout to satisfy the following properties:

F1. $\quad F(x, u, u)=f(x, u)$.

F2a. $u \rightarrow F(x, u, v)$ is nonincreasing for all $x \in[0,1], \quad v, u \in \mathbb{R}$.

F2b. $\quad v \rightarrow F(x, u, v)$ is nondecreasing for all $x \in[0,1], \quad u, v \in \mathbb{R}$.

F3. $\quad F(x, u, v)$ is Lipschitz continuous in $x, u, v{ }^{2}$

We now give three examples of numerical flux functions that satisfy the properties above.

1) Lax-Friedrichs [7]:

$$
F(x, u, v)=\frac{1}{2}\{f(x, u)+f(x, v)-\lambda(u-v)\},
$$

where $\lambda \geqq\|(\partial / \partial u) f(x, u)\|_{\infty}$.

2) Godunov [10]:

$$
F(x, u, v)= \begin{cases}\max _{u \leqq s \leqq v} f(x, s) & \text { if } u \leqq v \\ \min _{v \leqq s \leqq u} f(x, s) & \text { if } v \leqq u .\end{cases}
$$

3) Engquist-Osher [1], [11].

$$
F(x, u, v)=\int_{0}^{u} \min \left(\frac{\partial}{\partial s} f(x, s), 0\right) d s+\int_{0}^{v} \max \left(\frac{\partial}{\partial s} f(x, s), 0\right) d s+f(x, 0) .
$$

The following theorem is a straightforward extension of known results [1], [8].

\footnotetext{
${ }^{2}$ Assumption F3 need only be valid in the a priori interval determined by the maximum principle.
} 
THEOREM 4.1. Under properties $\mathrm{F} 1, \mathrm{~F} 2$, and $\mathrm{F} 3$, and condition (1.2) of $\S 1$, the difference scheme (4.1) has a unique solution for every grid. Moreover, there exists a constant $c$ independent of the grid such that

$$
\left\|v_{h}\right\|_{\infty}+\operatorname{var}\left(v_{h}\right) \leqq c .
$$

By using the results of Theorem 4.1 we next prove the following.

THEOREM 4.2. Under the conditions of the previous theorem, the family of approximations $\left\{v_{h}\right\}$ satisfy the AEI; consequently by Theorem 2.1 they satisfy the rate of convergence

$$
\left\|v_{h}-\bar{u}\right\|_{1} \leqq c \sqrt{h} \text {, }
$$

where $h=\max \Delta x_{j}, \bar{u}=\lim _{\varepsilon \downarrow 0} u_{\varepsilon}$ and for some constant $c$ which does not depend on $h$.

Proof of Theorem 4.2. For arbitrary $k \in \mathbb{R}, \phi \in C_{+}^{\infty}$ and $0 \leqq \alpha, \beta \leqq 1$, we estimate the quantity

$$
\begin{aligned}
\int_{0}^{1} \operatorname{sgn}\left(v_{h}-k\right) & \left\{-\left(f\left(x, v_{h}\right)-f(x, k)\right) \phi_{x}+\left(b\left(x, v_{h}\right)+\frac{\partial}{\partial x} f(x, k)\right) \phi\right\} d x \\
+ & \operatorname{sgn}\left(\gamma_{1}-k\right)\left\{f\left(\beta, v_{h}(\beta)\right)-f(1, k)\right\} \phi(1) \\
& -\operatorname{sgn}\left(\gamma_{0}-k\right)\left\{f\left(\alpha, v_{h}(\alpha)\right)-f(0, k)\right\} \phi(0),
\end{aligned}
$$

where $v_{h}$ is the piecewise constant interpolation of grid values generated by (4.1). Using the explicit form of $v_{h}$ and integration by parts we find that the integral term in (4.3) is given by

$$
\begin{array}{r}
\sum_{j=0}^{J-1} \operatorname{sgn}\left(u_{j}-k\right)\left\{-\left(f\left(x_{j+1}, u_{j}\right)-f\left(x_{j+1}, k\right)\right) \phi\left(x_{j+1}\right)+\left(f\left(x_{j}, u_{j}\right)-f\left(x_{j}, k\right)\right) \phi\left(x_{j}\right)\right. \\
\left.+\int_{x_{j}}^{x_{j+1}}\left(\frac{\partial}{\partial x} f\left(x, u_{j}\right)+b\left(x, u_{j}\right)\right) \phi(x) d x\right\} .
\end{array}
$$

Rearranging terms and then adding and subtracting $F\left(x_{j+1}, u_{j+1}, u_{j}\right)$ and $F\left(x_{j}, u_{j}, u_{j-1}\right)$ into this result we get that (4.4) equals

$$
\begin{aligned}
\sum_{j=1}^{J} \operatorname{sgn}\left(u_{j-1}-\right. & k)\left[-\left(F\left(x_{j}, u_{j}, u_{j-1}\right)-f\left(x_{j}, k\right)\right)\right] \phi\left(x_{j}\right) \\
& +\sum_{j=0}^{J-1} \operatorname{sgn}\left(u_{j}-k\right)\left[\left(F\left(x_{j}, u_{j}, u_{j-1}\right)-f\left(x_{j}, k\right)\right)\right] \phi\left(x_{j}\right) \\
& +\sum_{j=0}^{J-1} \operatorname{sgn}\left(u_{j}-k\right)\left[\left(F\left(x_{j+1}, u_{j+1}, u_{j}\right)-f\left(x_{j+1}, u_{j}\right)\right)\right] \phi\left(x_{j+1}\right) \\
& +\sum_{j=0}^{J-1} \operatorname{sgn}\left(u_{j}-k\right)\left[-\left(F\left(x_{j}, u_{j}, u_{j-1}\right)-f\left(x_{j}, u_{j}\right)\right)\right] \phi\left(x_{j}\right) \\
& +\sum_{j=0}^{J-1} \operatorname{sgn}\left(u_{j}-k\right) \int_{x_{j}}^{x_{j+1}}\left(\frac{\partial}{\partial x} f\left(x, u_{j}\right)+b\left(x, u_{j}\right)\right) \phi(x) d x \\
= & \mathrm{I}+\mathrm{II}+\mathrm{III}+\mathrm{IV}+\mathrm{V} .
\end{aligned}
$$

Before proceeding we give two simple lemmas.

LEMMA 4.1. For any three numbers $a, b, k \in \mathbb{R}$ and $x \in[0,1]$ we have

$$
\{\operatorname{sgn}(b-k)-\operatorname{sgn}(a-k)\}\{F(x, b, a)-f(x, k)\} \leqq 0 .
$$

Proof. The quantity above can be written as

$$
\{\cdots\}\{F(x, b, a)-F(x, b, k)\}+\{\cdots\}\{F(x, b, k)-F(x, k, k)\},
$$


where $\{\cdots\}=\{\operatorname{sgn}(b-k)-\operatorname{sgn}(a-k)\}$ and where we have used property F1 to write $f(x, k)=F(x, k, k)$. We can now bound this above by

$$
\begin{aligned}
& |F(x, b, a)-F(x, b, k)|-\operatorname{sgn}(a-k)\{F(x, b, a)-F(x, b, k)\} \\
& \quad+\operatorname{sgn}(b-k)\{F(x, b, k)-F(x, k, k)\}+|F(x, b, k)-F(x, k, k)| .
\end{aligned}
$$

Finally, using properties F2a and F2b shows us that this quantity is equal to zero.

LEMMA 4.2. If $H(x) \in C^{1}, \phi(x) \in C^{1}$, then

$$
\begin{aligned}
& \left|\int_{x_{j}}^{x_{j+1}} H(x) \phi(x) d x-\Delta x_{j} H\left(x_{j}\right) \phi\left(x_{j}\right)\right| \\
& \quad \leqq \Delta x_{j}\left[\left\|\frac{d}{d x} H\right\|_{\infty} \int_{x_{j}}^{x_{j+1}}|\phi(s)| d s+\|H\|_{\infty} \int_{x_{j}}^{x_{j+1}}\left|\frac{d}{d s} \phi(s)\right| d s\right] .
\end{aligned}
$$

Proof. The left-hand side of the inequality above can be written as

$$
\left|\int_{x_{j}}^{x_{j+1}}\left[\left(H(x)-H\left(x_{j}\right)\right) \phi(x)+H\left(x_{j}\right)\left(\phi(x)-\phi\left(x_{j}\right)\right)\right] d x\right|,
$$

which is bounded above by

$$
\Delta x_{j}\left\|\frac{d}{d x} H\right\|_{\infty} \int_{x_{j}}^{x_{j+1}}|\phi(x)| d x+\|H\|_{\infty}\left|\int_{x_{j}}^{x_{j+1}} \int_{x_{j}}^{x} \frac{d}{d s} \phi(s) d s d x\right| .
$$

The final estimate is now obvious.

Continuing the proof of the theorem, it is clear with the result of Lemma 4.1 that the sums of terms I and II of (4.5) is bounded above by

$$
\begin{aligned}
\mathrm{I}+\mathrm{II} \leqq-\operatorname{sgn} & \left(u_{J-1}-k\right)\left\{F\left(1, \gamma_{1}, u_{J-1}\right)-f(1, k)\right\} \phi(1) \\
& +\operatorname{sgn}\left(u_{0}-k\right)\left\{F\left(0, u_{0}, \gamma_{0}\right)-f(0, k)\right\} \phi(0) .
\end{aligned}
$$

Moreover, the sum of III and IV can be rewritten as

$$
\begin{array}{r}
\sum_{j=0}^{J-1} \operatorname{sgn}\left(u_{j}-k\right)\left\{F\left(x_{j+1}, u_{j+1}, u_{j}\right)-f\left(x_{j+1}, u_{j}\right)\right\}\left(\phi\left(x_{j+1}\right)-\phi\left(x_{j}\right)\right) \\
+\sum_{j=0}^{J-1} \operatorname{sgn}\left(u_{j}-k\right)\left\{\left(F\left(x_{j+1}, u_{j+1}, u_{j}\right)-F\left(x_{j}, u_{j}, u_{j-1}\right)\right)\right. \\
\left.-\left(f\left(x_{j+1}, u_{j}\right)-f\left(x_{j}, u_{j}\right)\right)\right\} \phi\left(x_{j}\right),
\end{array}
$$

and using Lemma 4.2 we see that term $\mathrm{V}$ of (4.5) can be bounded above by

$$
\begin{aligned}
& \sum_{j=0}^{J-1} \operatorname{sgn}\left(u_{j}-k\right)\left\{\left(\frac{\partial}{\partial x} f\left(x_{j}, u_{j}\right)+b\left(x_{j}, u_{j}\right)\right) \Delta x_{j}\right\} \\
& +h\left(\left\|\frac{d}{d x} H\left(x, v_{h}\right)\right\|_{\infty}+\left\|H\left(x, v_{h}\right)\right\|_{\infty}\right) \int_{0}^{1}\left(|\phi(x)|+\left|\frac{d}{d x} \phi(x)\right|\right) d x,
\end{aligned}
$$

where $H(x, u)=(\partial / \partial x) f(x, u)+b(x, u)$ and $h=\max \Delta x_{j}$. Combining these estimates and using the difference scheme (4.1) we conclude that

$$
\begin{aligned}
& \int_{0}^{1} \operatorname{sgn}\left(v_{h}-k\right)\left\{-\left(f\left(x, v_{h}\right)-f(x, k)\right) \phi_{x}+\left(b\left(x, v_{h}\right)+\frac{\partial}{\partial x} f(x, k)\right) \phi\right\} d x \\
& \leqq-\operatorname{sgn}\left(u_{J-1}-k\right)\left\{F\left(1, \gamma_{1}, u_{J-1}\right)-f(1, k)\right\} \phi(1) \\
& +\operatorname{sgn}\left(u_{0}-k\right)\left\{F\left(0, u_{0}, \gamma_{0}\right)-f(0, k)\right\} \phi(0) \\
& \quad+\sum_{j=0}^{J-1} L\left|u_{j+1}-u_{j}\right| \int_{x_{j}}^{x_{j+1}}\left|\phi_{x}\right| d x+h \text { const. }\left\{\int_{0}^{1}\left(|\phi|+\left|\phi_{x}\right|\right) d x\right\},
\end{aligned}
$$


where $L$ is the Lipschitz constant, given by

$$
L=\sup \left\{\frac{|F(x, u, v)-F(x, v, v)|}{|u-v|}, u, v \in I, x \in[0,1]\right\} .
$$

Next we include the boundary terms of the AEI into the inequality above. Using the fact that Lemma 4.1 implies that

$$
-\operatorname{sgn}\left(u_{J-1}-k\right)\left\{F\left(1, \gamma_{1}, u_{J-1}\right)-f(1, k)\right\} \leqq-\operatorname{sgn}\left(\gamma_{1}-k\right)\left\{F\left(1, \gamma_{1}, u_{J-1}\right)-f(1, k)\right\}
$$

and

$$
\operatorname{sgn}\left(u_{0}-k\right)\left\{F\left(0, u_{0}, \gamma_{0}\right)-f(0, k)\right\} \leqq \operatorname{sgn}\left(\gamma_{0}-k\right)\left\{F\left(0, u_{0}, \gamma_{0}\right)-f(0, k)\right\},
$$

we find that for $\alpha, \beta \in(0,1)$

$$
\begin{aligned}
& \int_{0}^{1} \operatorname{sgn}\left(v_{h}-k\right)\left\{-\left(f\left(x, v_{h}\right)-f(x, k)\right) \phi_{x}+\left(b\left(x, v_{h}\right)+\frac{\partial}{\partial x} f(x, k)\right) \phi\right\} d x \\
& \quad+\operatorname{sgn}\left(\gamma_{1}-k\right)\left\{f\left(\beta, v_{h}(\beta)\right)-f(1, k)\right\} \phi(1) \\
& \quad-\operatorname{sgn}\left(\gamma_{0}-k\right)\left\{f\left(\alpha, v_{h}(\alpha)\right)-f(0, k)\right\} \phi(0)
\end{aligned}
$$

is bounded above by

$$
\begin{aligned}
\operatorname{sgn}\left(\gamma_{1}-k\right) & \left\{f\left(\beta, v_{h}(\beta)\right)-F\left(1, \gamma_{1}, u_{J-1}\right)\right\} \phi(1) \\
& -\operatorname{sgn}\left(\gamma_{0}-k\right)\left\{f\left(\alpha, v_{h}(\alpha)\right)-F\left(0, u_{0}, \gamma_{0}\right)\right\} \phi(0) \\
& +L \sum_{j=0}^{J-1}\left|u_{j+1}-u_{j}\right| \int_{x_{j}}^{x_{j+1}}\left|\phi_{x}\right| d x+h \text { const. }\left\{\int_{0}^{1}\left(|\phi|+\left|\phi_{x}\right|\right) d x\right\} .
\end{aligned}
$$

We estimate the first boundary term above only since the second can be estimated in a similar way. Sum (4.1) from $j=j_{1}$ to $J-1$ where $j_{1}$ is chosen so that $x_{j_{1}} \leqq \beta<x_{j_{1}+1}$. Doing so, we substitute the result into the first term of (4.8) and find that

$$
\begin{aligned}
\operatorname{sgn}\left(\gamma_{1}-k\right) & \left\{f\left(\beta, v_{h}(\beta)\right)-F\left(1, \gamma_{1}, u_{J-1}\right)\right\} \\
& \leqq L\left|u_{j_{1}}-u_{j_{1}-1}\right|+\left\|\frac{\partial}{\partial x} f(x, u)\right\|_{\infty}\left|\beta-x_{j_{1}}\right|+\sum_{j=j_{1}}^{j-1}\left|B\left(x_{j}, x_{j+1}, u_{j}\right)\right| \Delta x_{j} \\
& \leqq L\left|u_{j_{1}}-u_{j_{1}-1}\right|+\text { const. }(h+1-\beta) .
\end{aligned}
$$

Now define

$$
H_{h}(x)=\sum_{j=0}^{J-1} \frac{\left|u_{j+1}-u_{j}\right|}{\Delta x_{j}} \chi_{I_{j}}(x)
$$

and note that

$$
\int_{0}^{1} H_{h}(x) d x=\sum_{j=0}^{J-1}\left|u_{j+1}-u_{j}\right| \leqq \operatorname{var}\left(v_{h}\right)
$$

Inserting this and the estimate above into (4.8) we have that (4.7) is bounded above by

$$
\text { const. }\left\{\left(h H_{h}(\beta)+1-\beta+h\right) \phi(1)+\left(h H_{h}(\alpha)+\alpha+h\right) \phi(0)+h \int_{0}^{1}\left(H_{h}(x)+1\right)\left|\phi_{x}\right| d x\right\} \text {. }
$$

Note that above we have used the fact that

$$
\int_{0}^{1}|\phi(x)| d x \leqq \int_{0}^{1}\left|\phi_{x}(x)\right| d x+\phi(0) .
$$


Reading off the terms $R_{h}^{0}(\alpha), R_{h}^{1}(\beta)$ and $R_{h}^{2}(x)$ from above one easily establishes that they satisfy properties A0, A1 and A2 of Definition 2.1. Therefore the families of approximate solutions generated by finite difference schemes of the form (4.1) satisfy the AEI of Definition 2.1. This completes the proof of Theorem 4.2.

\section{REFERENCES}

[1] L. ABRAhAmSSON AND S. Osher, Monotone difference schemes for singular perturbation problems, SIAM J. Numer. Anal., 19 (1982), pp. 979-992.

[2] C. BARDOS, A. Y. LeRoux AND J. C. NeDElEC, First order quasilinear equations with boundary conditions, Comm. Partial Differential Equations, 4 (1979), pp. 1017-1034.

[3] P. BÉnilan ANd H. Touré, Sur l'équation générale $u_{t}=\phi(u)_{x x}-\psi(u)_{x}+v$, C.R. Acad. Sci., Paris, Sér. I Math., 18 (1984), pp. 919-922.

[4] F. A. Howes, Boundary-interior layer interactions in nonlinear singular perturbation theory, Mem. Amer. Math. Soc., 203 (1978).

[5] S. N. KRUZKOV, First order quasi-linear equations in several independent variables, Math. USSR-Sb., 10 (1970), pp. 217-243.

[6] N. N. KUZNETSOV, On stable methods for nonlinear first order partial differential equations in the class of discontinuous functions, Proc. Roy. Irish Acad. Conf. Numer. Anal. (1976), pp. 183-197.

[7] P. D. LAX, Weak solutions of nonlinear hyperbolic equations and their numerical computation, Comm. Pure Appl. Math., 7 (1954), pp. 159-193.

[8] J. LORENZ, Nonlinear boundary value problems with turning points and properties of difference schemes, in Lecture Notes in Mathematics 942, W. Eckhaus and E. M. de Jager, eds., Springer-Verlag, Berlin, 1982.

[9] J. LORENZ AND R. SANDERS, Second order nonlinear singular perturbation problems with boundary conditions of mixed type, this Journal, 17 (1986), pp. 580-594.

[10] O. A. Oleinik, Discontinuous solutions of nonlinear differential equations, Amer. Math. Soc. Transl., 26 (1962), pp. 95-172.

[11] S. OsHER, Nonlinear singular perturbation problems and one-sided difference schemes, SIAM J. Numer. Anal., 18 (1981), pp. 129-144.

[12] B. PERTHAME AND R. SANDERS, The Neumann problem for nonlinear second order singular perturbation problems, this Journal, to appear.

[13] R. SANDERS, On convergence of monotone finite difference schemes with variable spatial differencing, Math. Comp., 40 (1983), pp. 91-106.

[14] A. I. VOL'PERT, The spaces BV and quasilinear equations, Math. USSR-Sb., 2 (1967), pp. 225-267. 